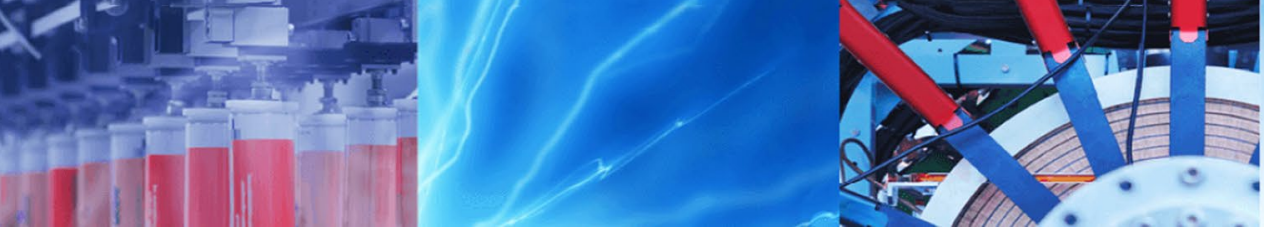

Research Article

\title{
Backstepping LQG controller design for stabilizing and trajectory tracking of vehicle suspension system
}

\author{
Akshaya Kumar Patra ${ }^{1}$
}

Received: 17 May 2019 / Accepted: 3 January 2020 / Published online: 10 January 2020

(c) Springer Nature Switzerland AG 2020

\begin{abstract}
The aim of this paper is to design a backstepping linear quadratic Gaussian controller (BLQGC) for a vehicle suspension (VS) system to improve the ride comfort by absorbing the shocks due to a rough and uneven road. For designing of the BLQGC, a forth order linearized model of the VS system is considered. In this control strategy, the conventional linear quadratic regulator is re-formulated with a state estimator based on the backstepping control approach to improve the control performance. The validation of improved control performance of BLQGC is established by comparative result investigation with other published control algorithms. The comparative results clearly reveal the better performance of the proposed approach to control the oscillation of the VS system within a stable range in terms of accuracy, robustness and handling uncertainties.
\end{abstract}

Keywords Ride comfort · Road profile ·Vehicle suspension system · Backstepping control · LQR

\section{Introduction}

In recent times, many researchers in the field of vehicle suspension (VS) system dynamics have devoted to arrive at an optimal solution with a compromise between vehicle handling, ride comfort and stability. There is a need for better approach today as the above problems are very much evident in the modern vehicle cases. Specific to the large sedan and luxury cars, even if excellent ride qualities are achieved, it is limited to acquiring adequate handling behaviour. With reference to sports vehicles, although it is provided with very good handling capability, but fails to provide desirable ride quality. There are many options in between for variations in the designing stage of the vehicle manufacturers to meet the customer needs. Designing point of view, passenger comfort and vehicle control are the two primary objectives to be considered. Road disturbances such as bumps or potholes are to be handled not to sacrifice the passengers comfort. At the control stage these factors are generally considered either through keeping the vehicle body from rolling and pitching excessively, or by maintaining good contact between the tire and the road.

Now a day's hydraulic dampers (shock absorbers) and springs are extensively used for vehicle suspensions. As a principle, these are charged with the job of absorbing bumps, minimizing the vehicle's body motions during acceleration, braking and turning of the vehicle, and keeping the tires in contact with the road surface. However, from the design point view these objectives are contradictory to each other to achieve all simultaneously at their optimum level.

The spring and the damper are the two essential components in VS design. The spring design mostly depends on the weight of the vehicle. The damper design is based on the suspensions placement on the compromise curve and so it is essential to be perfectly chosen to make the optimal vehicle performance for any type of vehicle. For

Akshaya Kumar Patra, akshayapatra@soa.ac.in; hiakp@yahoo.com | ${ }^{1}$ Department of Electrical and Electronics Engineering, ITER, Siksha 'O'Anusandhan University, Bhubaneswar, Odisha 751030, India. 
ideal performance, the damper should act such that passengers isolate from low-frequency road disturbances and absorb high frequency road disturbances. High damping is essential to achieve for best isolation of passengers from low-frequency disturbances.

However, even though it is desirable to design a high damping system, it is on the other degrade the high frequency absorption rate. In other way, providing low damping the damper offers adequate high-frequency absorption in terms of scarifying low-frequency isolation. To meet these contradictory objectives, it is essential to design and focus on automotive suspensions without compromising any of the factors mentioned above. As a solution to the above, three types of suspensions can be improved. These are passive, fully active, and semi-active type of suspensions. The spring and damper are the two basic components of the conventional passive suspension. Both the components are considered and fixed at the design stage. The suspension stores energy in the spring. Later it dissipates energy through the damper.

It is very much needed for further research to develop robust control algorithms to enhance the performance of VS system. This in turn enhances the vehicle capabilities to handle the aforementioned issues. An ideal design of suspension system needs to achieve many performance characteristics such as: (1) control of body movement (2) control of suspension movement and force distribution. Performance point of view the suspension should able to isolate the body for comfort against the road impact and inertial disturbances. These are generally associated with cornering and braking or acceleration of the vehicle system [1]. As discussed above, many performance objectives are conflicting in nature, that to enhance one the other factor degrades. Considering all the objectives, as a goal for the designing of a suspension system is difficult to meet [2]. Minimisation of vertical force to the passengers can be achieved by minimizing the vertical vehicles body acceleration of the suspension. Another factor which plays a vital role in passengers comfort is the optimal contact between wheel and road surface and this is essential in various driving conditions in order to maximize safety factor [3]. Among few design in the past, the system presented in [4] based on unconstrained optimizations for passive suspension (PS) system case is widely accepted and used. This successfully performs to achieve the desirability of low suspension stiffness, reduced unsprung mass, and an optimum damping ratio for the better controllability. As the PS system performs satisfactorily some extent, it is considered in many applications for the VS system. However, both the suspension spring and damper do not supply energy to the suspension system. They only control the motion of the vehicles body and wheel by limiting the suspension velocity. This is computed according to the rate specified by the designer. To overcome this issue, the Active Suspension (AS) system is considered as an efficient option for this application. The AS systems have the ability to dynamically respond to changes in the road profile. It is due to this fact that it can supply energy to produce relative motion between the body and the wheel. Sensors are provided in suspension system to measure the parameters dynamically. The parameters such as body velocity, suspension displacement, and wheel velocity and wheel and body acceleration are sensed and computed for the controller as input parameters [5]. An AS can be thought as the integration of the passive components to actuators that supply additional forces. These additional forces are computed by a feedback control law based on the input data from the sensors fixed to the vehicle. For modelling the real time dynamic conditions, the uncertainties due to system design and other external disturbances are needed to be considered for the controller. This motivates for a better control design to increase the robustness and controllability under uncertainties and disturbances.

During the past three decades, so many control strategy techniques are suggested and tested by absorbing the shocks due to a rough and bumpy road in case of VS system. Time-discrete and switching PID control strategy is implemented in VS problems with variable control gains based on the measured suspension variables $[6,7]$. However, the optimal gain parameter setting, a lesser range of robust control and need of change of gain setting with varying conditions are the major limitations to limit the real time application of these controllers. Among other projected robust control algorithms applied for limiting the oscillation and velocity of the VS system are fuzzy-logic control [8-13], fuzzy-PID control [14], genetic algorithm [15], neural network [16], neuro-fuzzy (NF) control [17], linear quadratic regulator (LQR) [18], $\mathrm{H}$-infinity $(H \infty)$ control [19, 20], and sliding mode (SM) control [21-23]. However, even if these control techniques are implemented effectively by absorbing the shocks due to the rough and bumpy road in case of VS system with enhanced accuracy and damping of oscillation, still fail to handle various constraints and random change found in a suspension environment. These control algorithms are not fully insensitive to the disturbances and the uncertainties of the model in spite of the improved performance. Hence, optimal control parameters setting for still better performance and for avoiding slow response following road disturbance (road impact), the current work suggests an alternative novel hybrid technique based on the backstepping control and LQR concept.

In this study, a hybrid concept based on backstepping control approach and principles of linear quadratic regulator is projected with the title backstepping linear quadratic Gaussian controller (BLQGC). The hybrid approach concept 
leads to an enhanced control performance with respect to robustness and time delay compensation characteristics to counteract the negative impact of associated errors, disturbances and uncertainties. The novel idea followed in this formulation is to modify LQR with a state estimator according to the approach of backstepping. The states of the VS system are estimated recursively based on the backstepping approach to improve the system performance. Secondly, a stabilizing control law is formulated by using the backstepping control approach [24-27]. Application of the suggested approach (BLQGC) to control the oscillation and velocity in vehicle suspension system results to ensure a high noise elimination capability, better capability to handle uncertainty, enhanced robustness, improved accuracy, and better stability in comparison to other well established approaches.

This manuscript is organized as follows. Section 2 concisely illustrates the VS model with mathematical details reflecting to its dynamic characteristics of its suspension vibration process. Also, it clearly demonstrates the simulation execution of the system on MATLAB environment. A detailed presentation on how the control technique is formulated and how it is implemented for this problem is presented in Sect. 3. Comparative results of the proposed approach with other published control techniques and the related analysis are provided in Sect. 4 . The concluding comments are summarized in the Sect. 5.

\section{Problem formulation and modeling}

\subsection{System overview}

The overall closed loop model of VS system is depicted in Fig. 1a. The road disturbance $w(t)$ and $v(t)$ are reflected as the process disturbance and the sensor noise respectively in this study. The controller receives information about the oscillation of the VS system as input to provide the optimal control force $u(t)$, and it is applied in-between the unsprung and the sprung mass to reduce the relative motion between them.

\subsection{Modelling of VS system}

In the year 1958, the concept of VS system was emerged into limelight. It has been found in the later period that the vibration suppression capability is limited in case of traditional PS and semi-active suspension systems. This leads to focus on an AS system with additional control forces as a better alternative to the above drawbacks to suppress the oscillations and used in modern vehicle industry. The major factor that differentiates the active suspension system performance wise is the ability to inject energy into vehicle dynamic system via actuators unlike dissipates energy in case of conventional suspension system. To produce a desirable control force to handle the variety of road disturbances in real time applications, actuators are placed in between the unsprung and the sprung mass in AS system. The most challenging task of designing an AS system is to enhance the ride comfort by absorbing the shocks due to the rough and bumpy road. The force actuator in the case of the AS system is capable to add and also dissipate energy from the system. This results an increase in the ride comfort and vehicle handing due to the capability of the suspension system to regulate the vehicle altitude and to eliminate the adverse effects of braking and vehicle roll during cornering and braking. The schematic model of the VS system is illustrated in Fig. $1 \mathrm{~b}$.

All the physical activities of the VS system are mathematically formulated and specified by Eqs. (1) and (2). All nomenclature and specifications for VS model are shown in Tables 1 and 2 respectively. The simulation model of the VS system is established based on the Eqs. (1) and (2) as displayed in the Fig. 1c.

$$
\begin{aligned}
m_{1}\left(\frac{d^{2} x_{1}(t)}{d t^{2}}\right)= & -b_{1}\left(\frac{d x_{1}(t)}{d t}-\frac{d x_{2}(t)}{d t}\right)-k_{1}\left(x_{1}(t)-x_{2}(t)\right)+u(t) \\
m_{2}\left(\frac{d^{2} x_{2}(t)}{d t^{2}}\right)= & b_{1}\left(\frac{d x_{1}(t)}{d t}-\frac{d x_{2}(t)}{d t}\right)+k_{1}\left(x_{1}(t)-x_{2}(t)\right) \\
& +b_{2}\left(\frac{d w(t)}{d t}-\frac{d x_{2}(t)}{d t}\right)+k_{2}\left(w(t)-x_{2}(t)\right)-u(t)
\end{aligned}
$$

\subsection{Linearization of VS system}

Figure 1c shows the non-linear model of VS system, and its simplified form is shown in Fig. $1 \mathrm{~d}$. The state-space equation of the VS model with $w(t)$ and $v(t)$ can be expressed as [18]:

$\left.\begin{array}{l}\dot{x}_{m}(t)=A_{m} x_{m}(t)+B_{m} u(t)+B_{d} w(t) \\ y(t)=C_{m} x_{m}(t)+D_{m} u(t)+v(t)\end{array}\right\}$

wh e re $A_{m}=\left[\begin{array}{cccc}0 & 0 & 1 & 0 \\ 0 & 0 & 0 & 1 \\ -32.000 & 32.000 & -0.1 & 0.1 \\ 250.00 & -1812.5 & 1.1 & -48.0\end{array}\right]$; $B_{m}=\left[\begin{array}{c}0 \\ 0 \\ 0.0004 \\ -0.0031\end{array}\right] ; B_{d}=\left[\begin{array}{c}0 \\ 0 \\ 4.5455 \\ 1.8182\end{array}\right] ; C_{m}=\left[\begin{array}{llll}1 & -1 & 0 & 0\end{array}\right] ;$ and $D_{m}=0$.

The $w(t), x_{m}(t), v(t)$, and $y(t)$ are the road disturbance, state variable, sensor noise, and measured output of the VS system respectively. The state variable $x_{m}(t)$ comprised with vertical displacement of the sprung mass $x_{1}(t)$, 


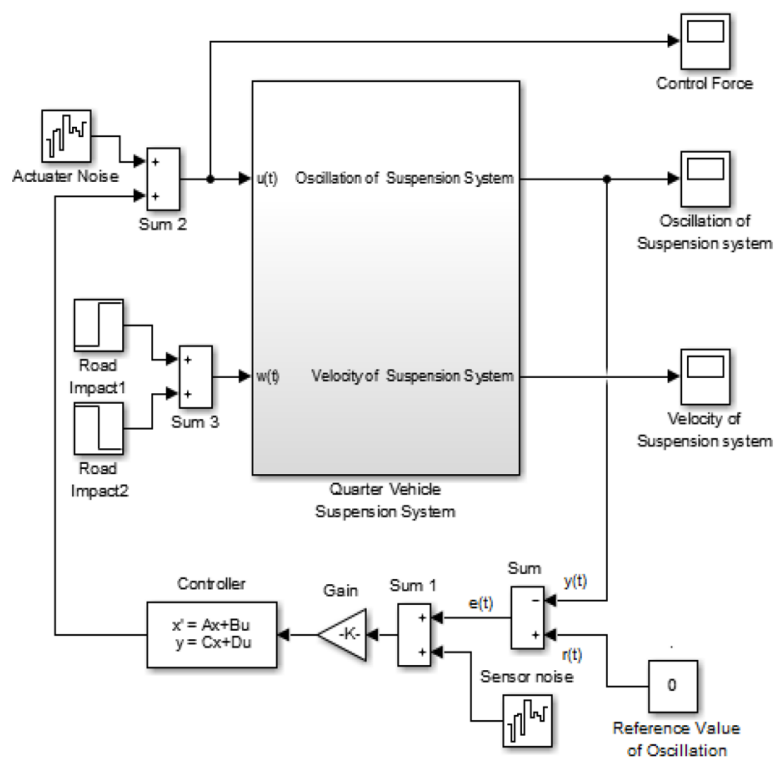

(a)

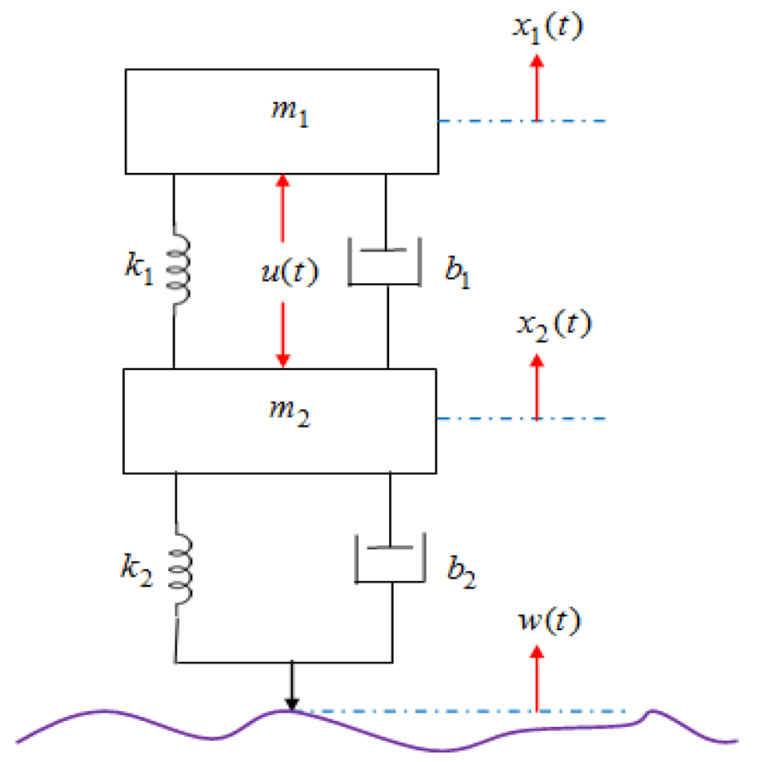

(b)

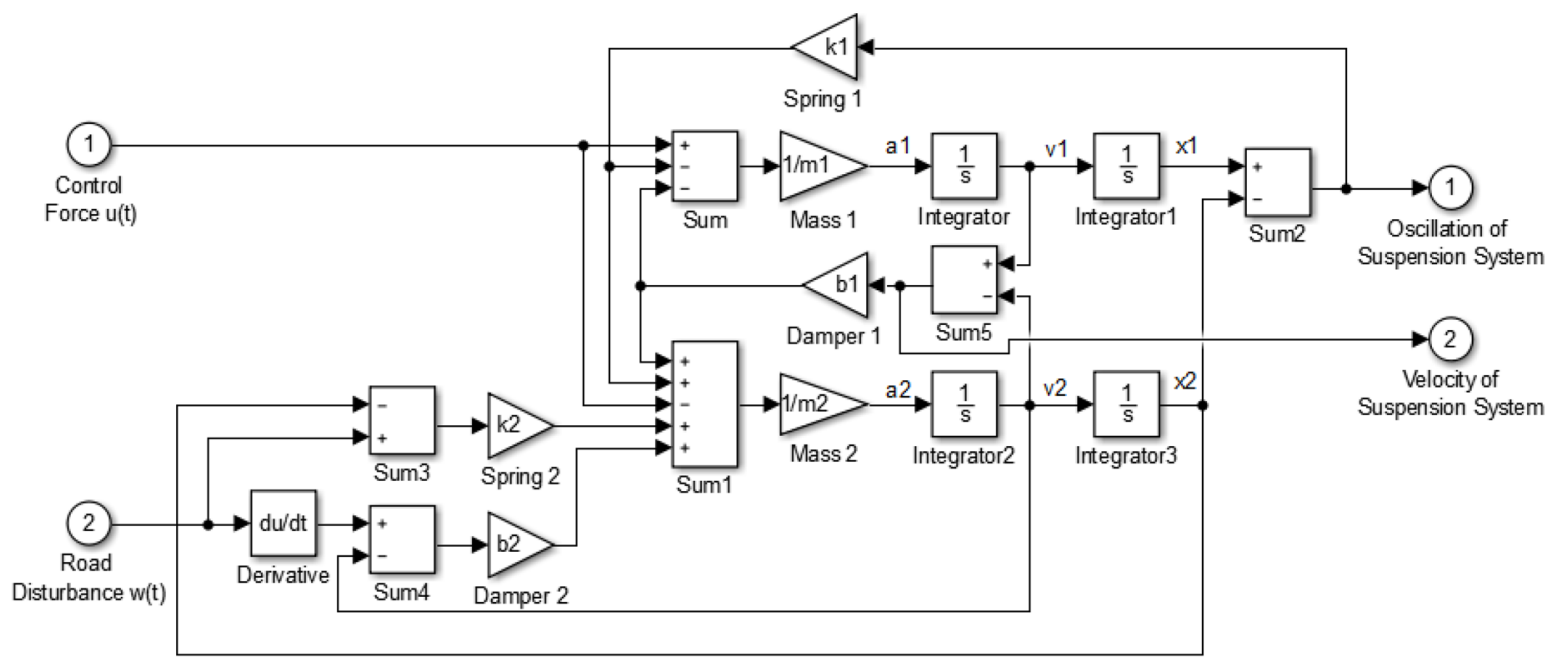

(c)

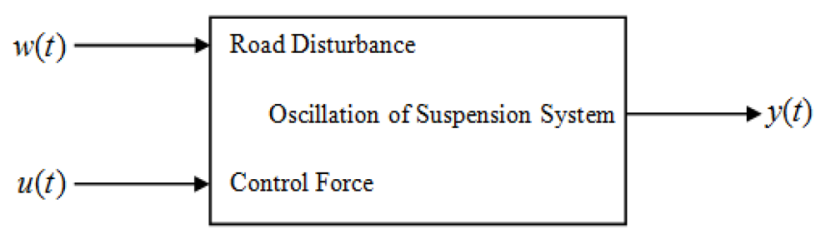

(d)

Fig. 1 a Closed loop VS model with BLQGC; b schematic model of the VS system; c simulation model of the non-linear VS system; $\mathbf{d}$ simplified diagram of VS system

vertical displacement of the unsprung mass $x_{2}(t)$, vertical velocity of the sprung mass $\dot{x}_{1}(t)$, and vertical velocity of the unsprung mass $\dot{x}_{2}(t)$. The $A_{m^{\prime}} B_{m^{\prime}} C_{m^{\prime}} D_{m}$ and
$B_{d}$ represent the matrices of the state-space model of VS system. The state-space matrices are found out from the linearization of the system dynamics nearby the reference 
Table 1 VS model states and parameters

\begin{tabular}{ll}
\hline Symbol & Description \\
\hline$w(t)$ & Road disturbance input acting on the unsprung mass \\
$x_{1}(t)$ & Displacement of the Sprung mass in vertical direction \\
$x_{2}(t)$ & Displacement of the unsprung mass in vertical direction \\
{$\left[x_{1}(t)-x_{2}(t)\right]$} & Oscillation of suspension system \\
$u(t)$ & Applied control force between the sprung and unsprung mass \\
\hline
\end{tabular}

Table 2 VS model specification values

\begin{tabular}{llrl}
\hline Symbol & Quantity/meaning & Value & Unit \\
\hline$m_{1}$ & $\begin{array}{c}\text { Sprung mass (mass of the vehicle sup- } \\
\text { ported by the suspension) }\end{array}$ & $2500 \mathrm{~kg}$ \\
$m_{2}$ & $\begin{array}{c}\text { Unsprung mass (mass of the axle and } \\
\text { wheel) }\end{array}$ & $320 \mathrm{~kg}$ \\
& $\begin{array}{l}\text { Spring constant of suspension system } \\
k_{1}\end{array}$ & $80,000 \mathrm{~N} / \mathrm{m}$ \\
$k_{2}$ & $\begin{array}{l}\text { Spring constant of wheel and tire } \\
b_{1}\end{array} \quad \begin{array}{r}\text { Damping coefficient of suspension } \\
\text { system }\end{array}$ & $300,000 \mathrm{~N} / \mathrm{m}$ \\
$b_{2}$ & Damping coefficient of wheel and tire & $15,020 \mathrm{~N} \mathrm{~s} / \mathrm{m}$ \\
\hline
\end{tabular}

point. The detailed derivation is described in the literature [18]. In the current study the initial reference such as the oscillation and the velocity of the suspension system are taken as zero. In MATLAB, the command 'linmod' is used to evaluate the state-space matrices on the VS model as depicted in Fig. 1c. The VS model is linearized surrounding the reference point for the design of robust controllers to control the oscillation and the velocity of the suspension system within the stable range.

\subsection{Performance analysis of VS system}

There are four poles in VS system. Out of them one pole lies in right hand side of the s-plane. As a result, the system becomes unstable. This needs the design of an adaptive controller for improving the stability of the system by means of shifting the poles into the left hand side of the s-plane. The VS system SIMULINK model in the open loop form is depicted in Fig. 1c. The VS system consists of two inputs and two outputs. The control force $u(t)$ and road disturbance (impact) $w(t)$ are the two inputs of the VS system. The oscillation and velocity of the suspension system are the two outputs of the VS system. An uncontrolled oscillation and velocity of the suspension system is being observed owing to the application of a vertical impulsive road disturbance of $0.1 \mathrm{~m}$ on the unsprung mass in case of VS system. The irregular oscillation and velocity of the suspension system are illustrated in Fig. 2a, b. Figure 2a, b illustrates the unstable dynamics under various model uncertainties and disturbances. These unstable dynamics can be reduced by applying the suitable control algorithms. In this case, the

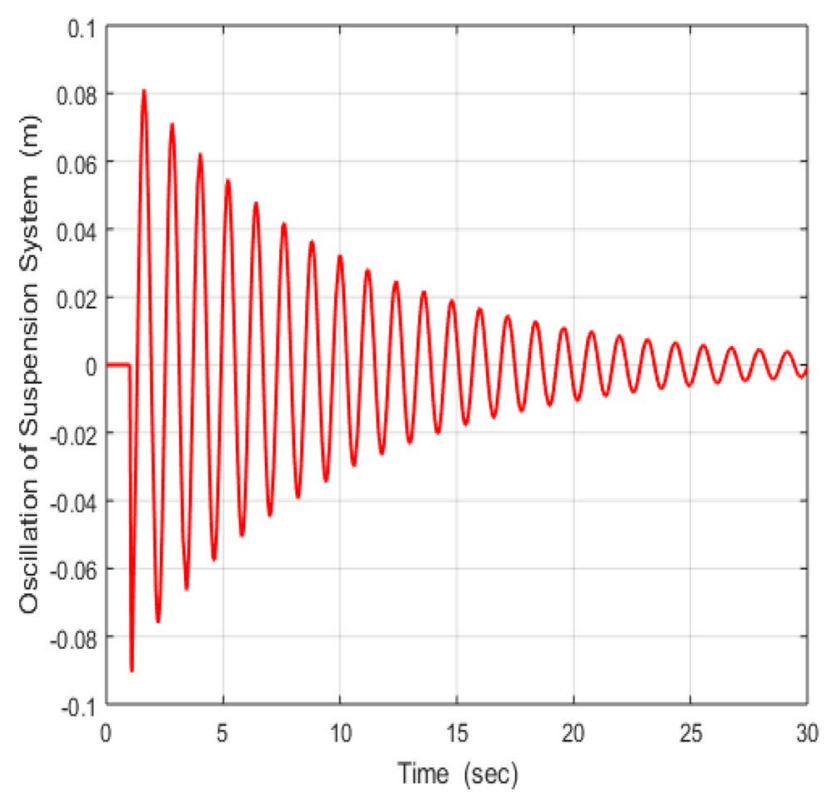

(a)

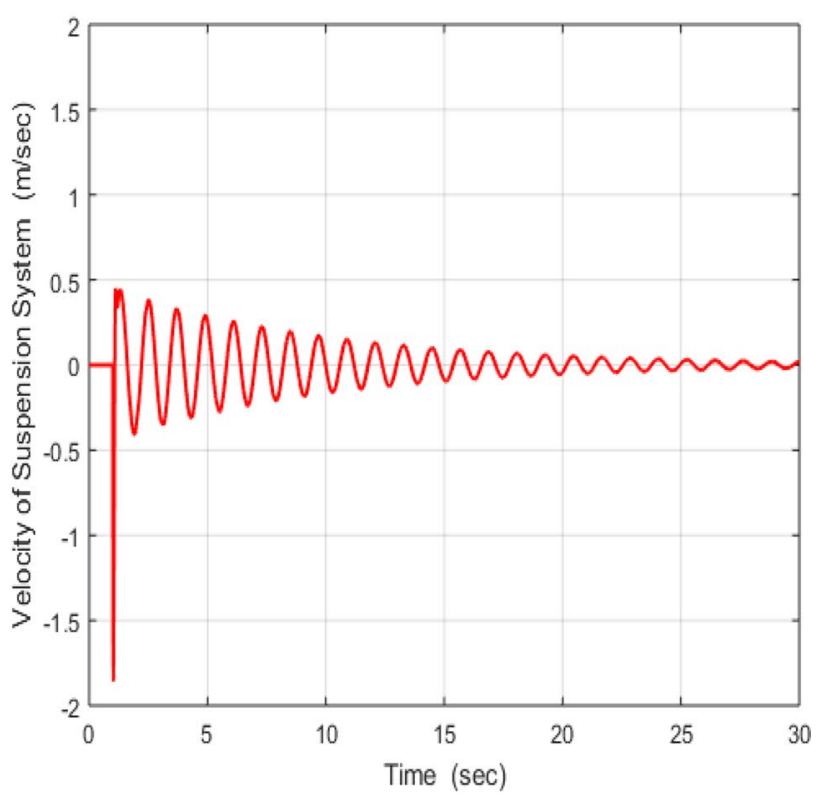

(b)

Fig. 2 a Response of oscillation of the suspension system with road impact; $\mathbf{b}$ response of velocity of the suspension system with road impact 
oscillation of the suspension system is the most essential outcome needs to be controlled within a stable range through suitable control techniques, and velocity of the suspension system is analyzed in order to view the motion trajectory.

\section{Control algorithms}

The BLQGC control algorithm is demonstrated in this section. The closed loop system response with respect to stability, accuracy and robustness are analyzed. The control specifications such as settling time (s), steady-state error (\%), overshoot $(\mathrm{m})$ and undershoot $(\mathrm{m})$ are also evaluated and examined with proper validation of the controller action.

\subsection{Design of BLQGC}

The linearized model of the VS system as discussed in Sect. 2.3 has been taken for the formulation of the suggested control algorithm to regulate the oscillation and the velocity of the suspension system. For accomplishing an upgraded performance and the adjustment of controller parameters of the suggested BLQGC, it is integrated to the linearized model of the VS system as illustrated in Fig. 3. The BLQGC is designed by integrating a state estimator with the LQR. The state estimator is utilized to pull all the states of the system towards the operating point to enhance the control action. The linearized model of the VS system with $w(t)$ and $v(t)$ is formulated as follows:

$$
\begin{aligned}
& \dot{x}_{m}(t)=A_{m} x_{m}(t)+B_{m} u(t)+B_{d} w(t) \\
& y(t)=C_{m} x_{m}(t)+v(t)
\end{aligned}
$$

The both $v(t)$ and $w(t)$ are the Gaussian noise vectors. The mathematical demonstrations are as:

$\left.\begin{array}{l}E\{w(t)\}=0 \\ E\{v(t)\}=0\end{array}\right\}$

$E\left\{w(t) w^{T}(\tau)\right\}=Q_{2} \delta(t-\tau)$

$E\left\{v(t) v^{T}(\tau)\right\}=R_{2} \delta(t-\tau)$

The both $w(t)$ and $v(t)$ are uncorrelated, therefore it can be defined as follows:

$E\left\{w(t) v^{T}(\tau)\right\}=0$

where $Q_{2}$ and $R_{2}$ are symbolized as the positive semi-definite intensity matrices of the $w(t)$ and $v(t)$ respectively. Figure 3 illustrates the linearized model of the VS system with the LQR gain $K_{c}$ and backstepping controller gain $K_{b}$. The computational methods of $K_{c}$ and $K_{b}$ are described in Sects. 3.1.1 and 3.1.2 respectively. The calculation of the Transfer Function (TF) of the BLQGC is described in Sect. 3.1.3.

\subsubsection{LQR gain $K_{c}$}

The $K_{c}$ is evaluated with respect to the VS system dynamics for the minimum value of the quadratic performance index $j$ as specified in the Eq. (10).

$$
j=\int_{0}^{\infty}\left[x_{m}(t)^{T} Q_{1} x_{m}(t)+u(t)^{T} R_{1} u(t)\right] d t
$$

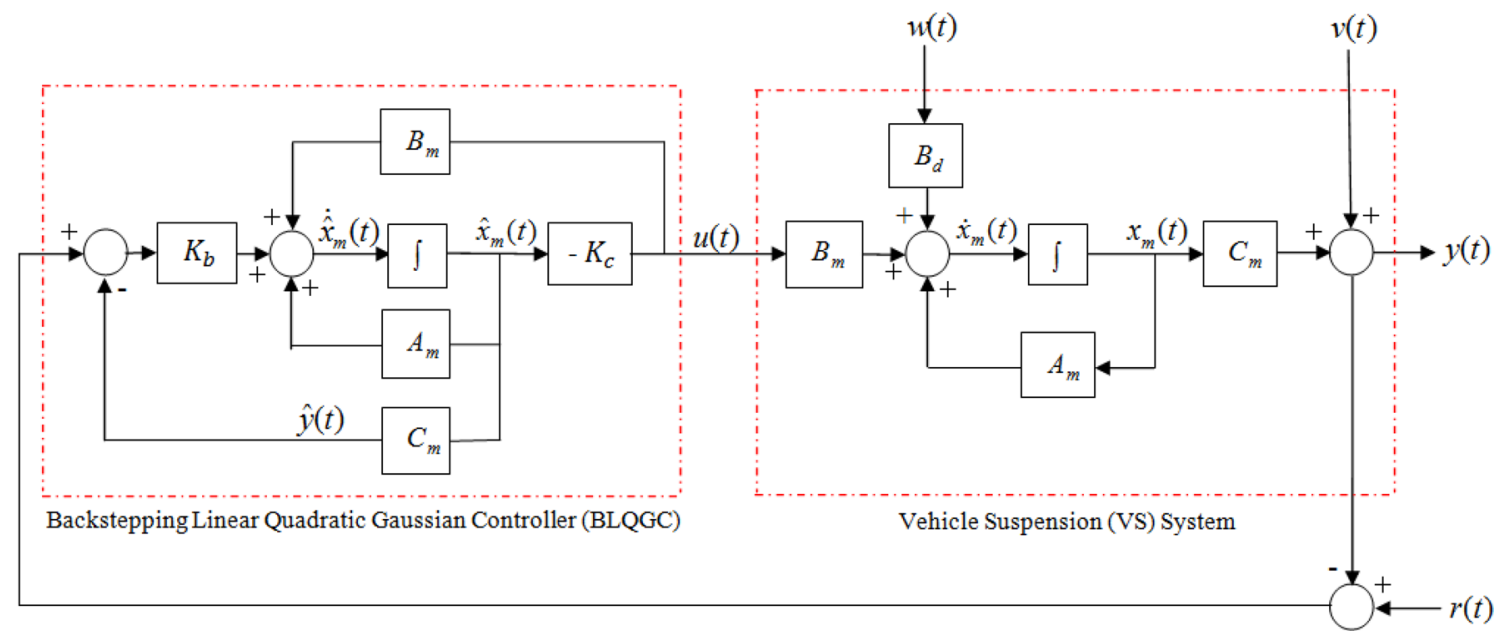

Fig. 3 Closed loop VS model with BLQGC in the state-space representation 
Fig. 4 The flow chart of proposed control algorithm (BLQGC)

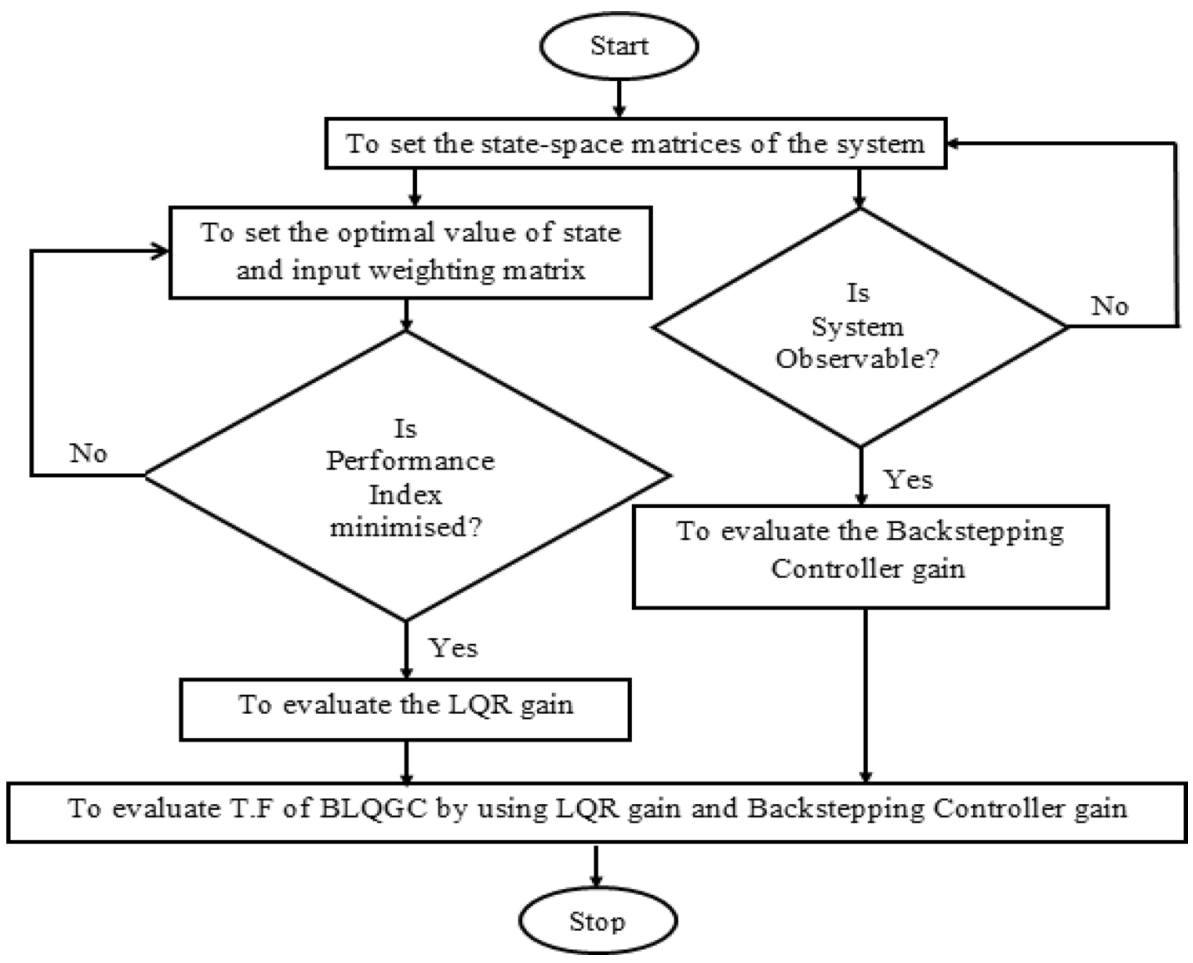

where $Q_{1}=C_{m}^{T} q_{1} C_{m}$. The $Q_{1}, q_{1}$, and $R_{1}$ are denoted as the positive semi-definite weighted matrix of state, the intensity of a weighted matrix of state, and the positive definite weighted matrix of input respectively. The $K_{c}$ is estimated for the minimum value of the quadratic performance index as specified in the Eq. (11).

$K_{c}=R_{1}^{-1} B_{m}^{T} \prod_{k}$

where $\prod_{k}$ is the solution of the Controller Algebraic Riccati Equation (CARE). The CARE is specified as follows [24]:

$A_{m}^{T} \prod_{k}+\prod_{k} A_{m}-\prod_{k} B_{m} R_{1}^{-1} B_{m}^{T} \prod_{k}+C_{m}^{T} q_{1} C_{m}=0$

\subsubsection{Backstepping controller gain $K_{b}$}

The states of the VS model are estimated recursively based on the backstepping approach to improve the system performance. The estimated state-space equation of the VS system is defined as:

$$
\begin{aligned}
& \frac{d \hat{x}_{m}(t)}{d t}=A_{m} \hat{x}_{m}(t)+B_{m} u(t)+K_{b}[r(t)-y(t)-\hat{y}(t)] \\
& \quad=\left(A_{m}-B_{m} K_{c}-K_{b} C_{m}\right) \hat{x}_{m}(t)+K_{b}[r(t)-y(t)] \\
& \hat{y}(t)=C_{m} \hat{x}_{m}(t)
\end{aligned}
$$

where $\hat{x}_{m}(t)$ and $\hat{y}(t)$ are denoted for the estimated state and estimated output respectively. The $K_{b}$ can be computed as follows [24]:

$K_{b}=\sum_{b} C_{m}^{T} R_{2}^{-1}$

where $\sum_{b}$ is the solution of the Lyapunov Equation (LE). The LE is specified as follows [24]:

$\sum_{b}\left(A_{m}-B_{m} K_{c}\right)+\left(A_{m}-B_{m} K_{c}\right)^{T} \sum_{b}+B_{d} Q_{2} B_{d}^{T}+I_{n}=0$

where $K_{c}$ is evaluated in such a manner that $\left(A_{m}-B_{m} K_{c}\right)$ is to be Hurwitz. The $I_{n}$ is an identity matrix with size of $n \times n$, and $n$ is the order of the matrix $A_{m}$.

\subsubsection{TF of BLQGC}

The TF of BLQGC, $K(s)$ is evaluated with the use of $K_{b}$ and $K_{c}$. The $K(s)$ of BLQGC is defined as:

$K(s)=K_{c}\left(s I_{n}-A_{m}+B_{m} K_{c}+K_{b} C_{m}\right)^{-1} K_{b}$

The packed matrix notation of $K(s)$ is specified as follows [24]:

$K(s)=\left[\begin{array}{c:c}A_{m}-B_{m} K_{c}-K_{b} C_{m} & K_{b} \\ \hdashline-K_{c} & 0\end{array}\right]$ 


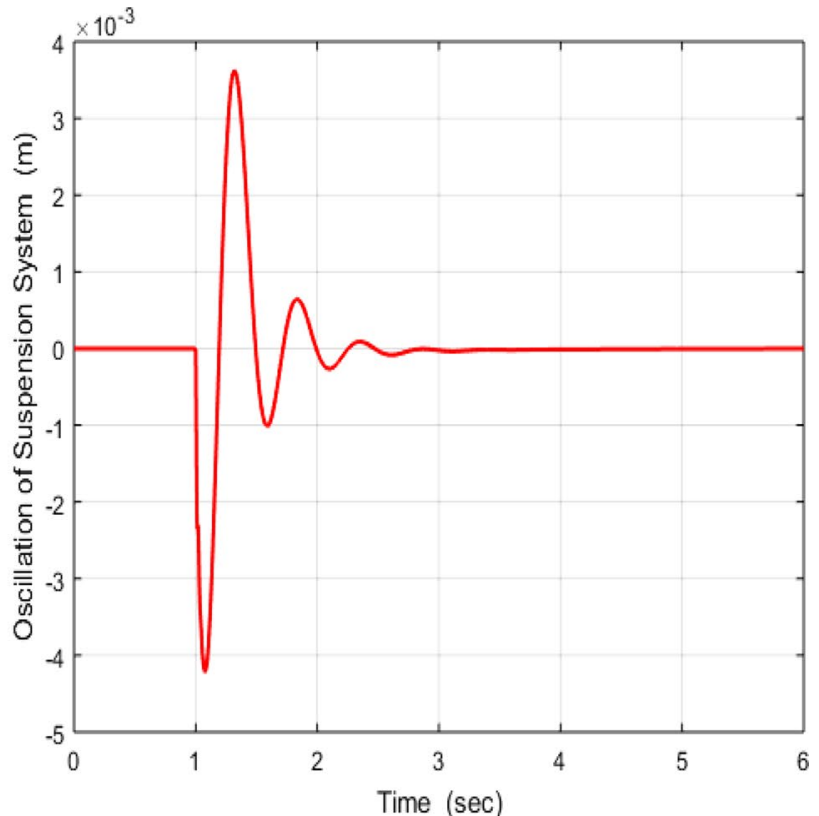

(a)

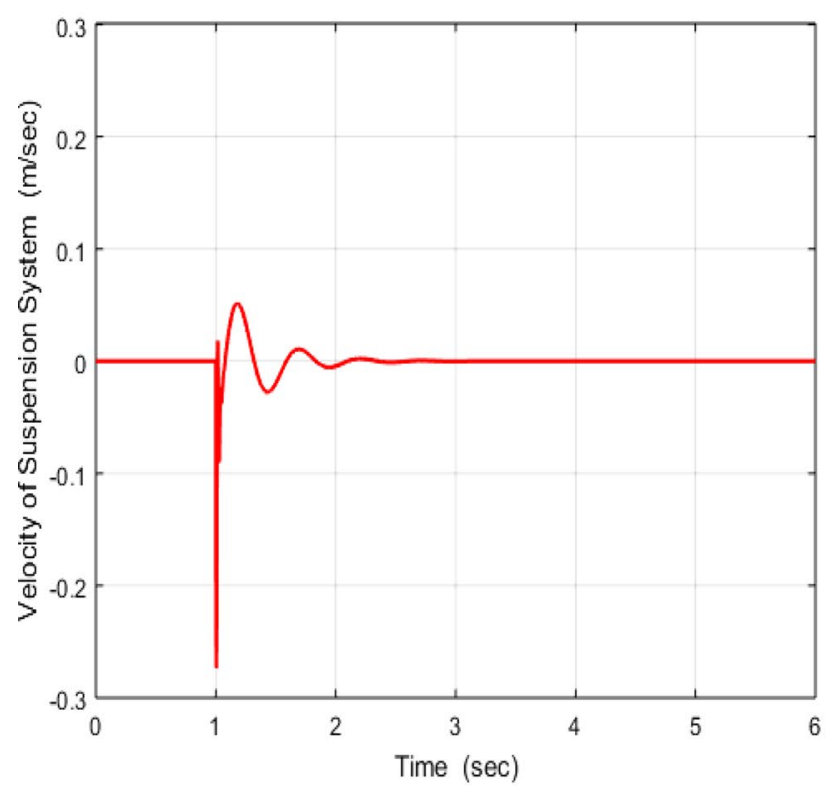

(b)

Fig. 5 a Response of oscillation of the suspension system with road disturbance based on BLQGC; $\mathbf{b}$ response of velocity of the suspension system with road disturbance based on BLQGC

The quadratic performance index $j$ is a time integral of the sum of transient energy and control energy stated as a function of time. Thus, to minimize that particular quadratic performance index $j$ by suitably selecting the state weighted matrix $Q_{1}$, input weighted matrix $R_{1}$ and to solve the Riccati equation subjected to system conditions in

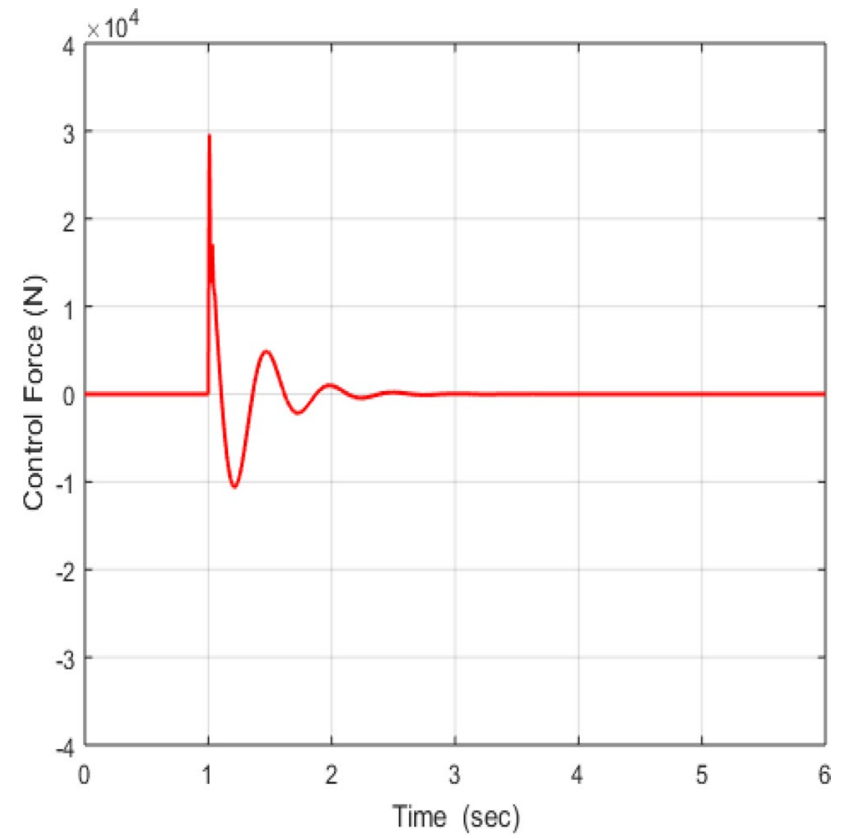

Fig. 6 Control force generated by BLQGC

order to determine the optimal control parameters. Two control parameters $R_{1}$ and $Q_{1}$ regulate the control input $u(t)$ and system outputs respectively. The controller is tuned by changing the intensity of $Q_{1}$ matrix to achieve the required output. The response of the uncontrolled process in Fig. $2 \mathrm{a}$ shows a larger effect of road impact in the oscillation of the suspension system. For controller design, thus maximum weightage is given to the states responsible for the oscillation of the suspension system. The weighted matrices and therefore chosen as $1000 * C_{m}^{T} * C_{m}, R_{1}=1, Q_{2}=0.01$, and $R_{2}=0.0025$. The entire control algorithm of BLQGC is also described through a flow chart as shown in Fig. 4.

\section{Result and discussions}

Time domain responses of oscillation and velocity, stability, and robustness of the closed loop VS system with proposed BLQGC are described in detail in this section. The proposed control approach is compared with other popular control algorithms to justify its enhanced performance.

\subsection{Performance analysis of VS system with BLQGC}

In this section, all physical activities of the closed loop VS model with suggested BLQGC are examined under different conditions and the huge deviation of road impact. The time domain response of the oscillation and the velocity of the suspension system with $0.1 \mathrm{~m}$ impulsive road 


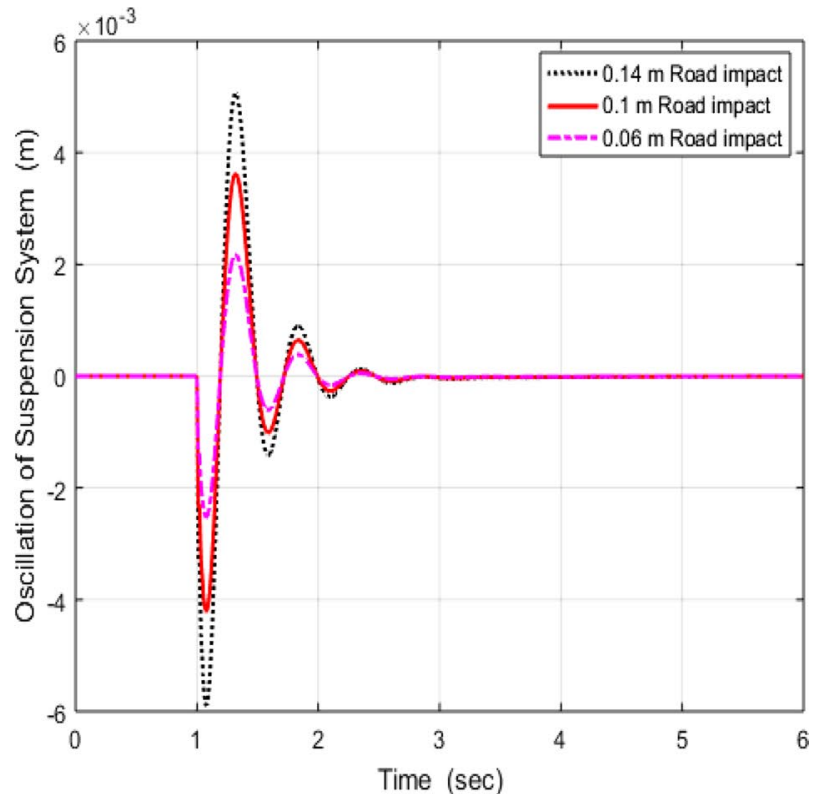

(a)

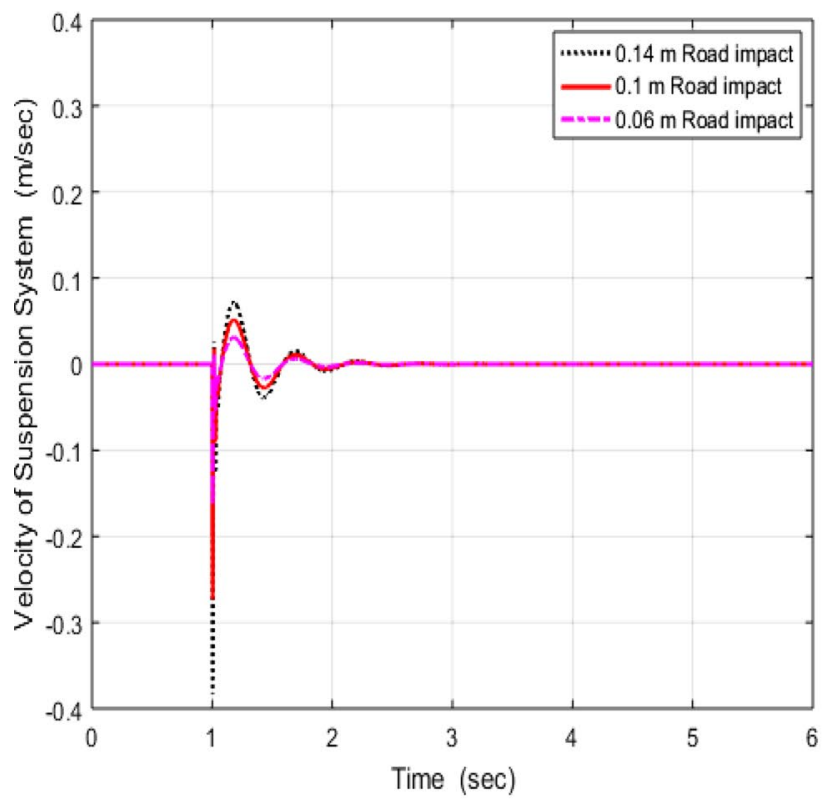

(b)

Fig. 7 a Oscillation of the suspension system with deviation of $\pm 40 \%$ road impact based on BLQGC; $\mathbf{b}$ velocity of the suspension system with deviation of $\pm 40 \%$ road impact based on BLQGC

disturbance (road impact) at the simulation time of $1.0 \mathrm{~s}$ is displayed in Fig. $5 \mathrm{a}$, b. The output results clearly specify the suspension system attains the zero oscillation and zero velocity with less settling time and also attains the balance position where the system is absolutely steady. To achieve the enhanced system response, the required control force

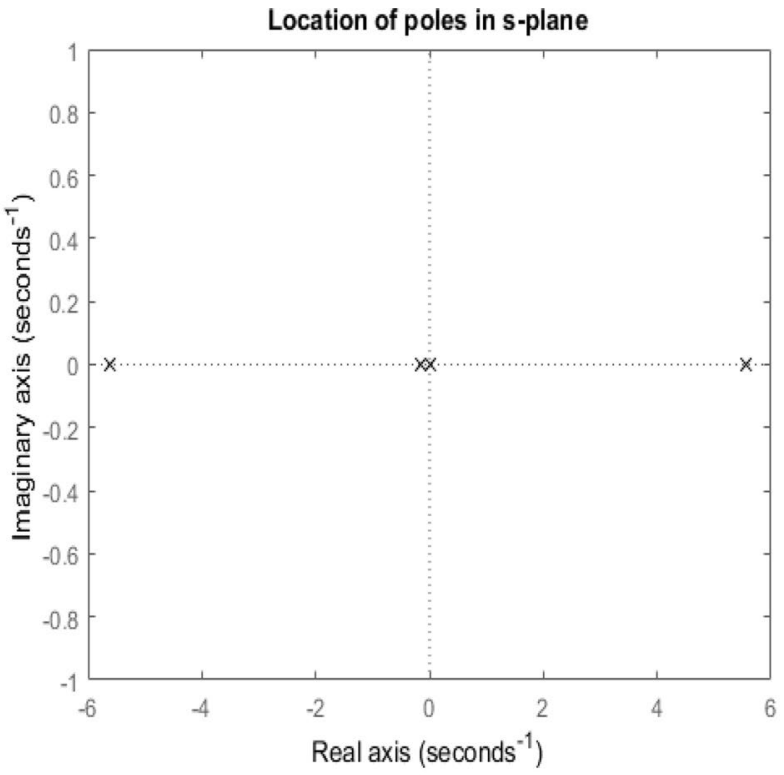

(a)

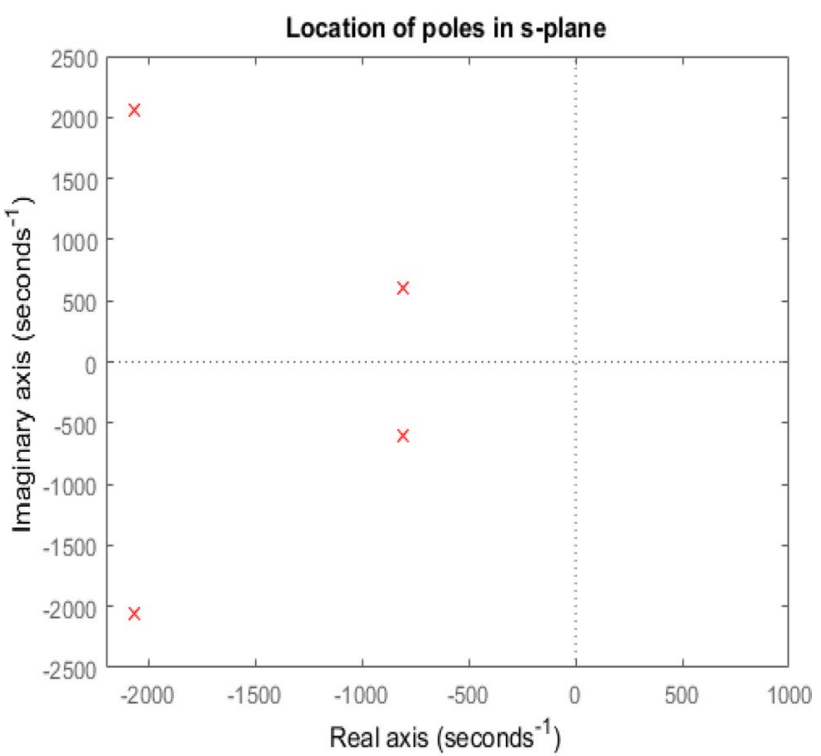

(b)

Fig. 8 a Location of VS system poles in s-plane; $\mathbf{b}$ location of closed loop VS system poles in the s-plane

$u(t)$ is generated by the suggested BLQGC and demonstrated in Fig. 6.

\subsection{Robustness of the BLQGC}

Figure $7 a, b$ illustrates the oscillation and the velocity of the VS system with suggested BLQGC under the huge deviation of road impact $w(t)$. The time domain outcomes under several road disturbances show the enhanced performance of the closed loop VS model with BLQGC. Overall 


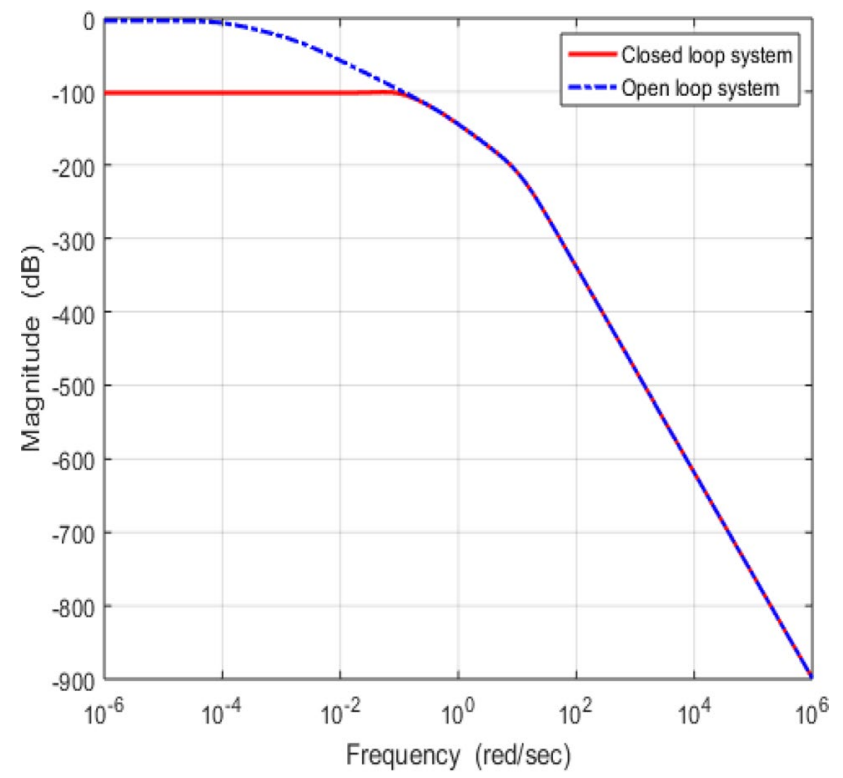

Fig. 9 The magnitude plot of the open and closed loop VS system

in each case, the VS system achieves finally zero oscillation and zero velocity with less settling time. As indicated by the results, the suggested controller's robust performance under the huge deviation of road disturbances compared to other published control techniques is much better.

\subsection{Stability analysis}

There are four poles in VS system. Out of them one pole is in the right hand side of the s-plane as displayed in Fig. 8 a. Therefore, the system is unstable. For enhancement of stability of the VS system, the BLQGC is developed and implemented in the VS system. As a result four poles of the VS system are dragged towards the left hand side of the $\mathrm{s}$-plane as displayed in Fig. 8b. Consequently the system stability is enhanced due to the suggested BLQGC.

Figure 9 illustrates the magnitude plots result of the open loop and closed loop VS system to verify and analyze the stability conditions. From both the magnitude plots, it is observed a better smoothness referring to the wider stead state stability of closed loop system than the open loop system. In other words the bandwidth is increased in case of a closed loop VS system with BLQGC than the open loop VS system. This clearly indicates a faster stable dynamics, and also it results zero oscillation and zero velocity with less settling time in case of the closed loop VS system. This justifies better stability during system operation.

\subsection{Comparative study}

The proposed BLQGC control approach is compared with other popular control approaches such as PID, fuzzy, NF, LQR, $H \infty$ and SM to justify its enhanced performance as a controller. Figure 5a illustrates the effect of road impact in the oscillation of the suspension system with the proposed BLQGC approach. Table 3 presents a comparative analysis with respect to settling time (s), peak overshoot $(\mathrm{m})$, peak undershoot $(\mathrm{m})$, noise $(\%)$, and steady state error $e_{s s}$. The effect of road impact in the oscillation of the suspension system applying different control approaches such as PID, fuzzy, NF, LQR, $H_{\infty}$ and SM is also presented in Table 3 based on the references $[6,11,17,18,20,23]$ respectively. Similar working conditions are followed with the same level of actuator and sensor noise in all control techniques application for comparison.

The oscillations of the suspension system under $0.1 \mathrm{~m}$ impulsive road disturbance are tested. The corresponding results are presented for the various control approaches along with the proposed BLQGC with respect to time domain specifications such as overshoots $(\mathrm{m})$, undershoots $(\mathrm{m})$ and settling time $(\mathrm{s})$. The results signify the better controllability of the BLQGC. The simulation results also demonstrate the high noise and chattering elimination capability with high robustness for the proposed approach. Overall, by looking to the above comparative analysis, the findings of suggested approach advantages are the higher accuracy and stability, more robustness, high noise elimination capability, and better capability to handle uncertainty under various conditions and huge variation of road impact.
Table 3 Comparative result analysis related to oscillation of the suspension system

\begin{tabular}{llllllll}
\hline Controller & PID [6] & Fuzzy [11] & NF [17] & LQR [18] & $H_{\infty}[20]$ & SM [23] & BLQGC (proposed) \\
\hline Road disturbance (m) & 0.1 & 0.1 & 0.1 & 0.1 & 0.1 & 0.1 & 0.1 \\
Settling time (s) & 4.2 & 4.1 & 3.7 & 3.4 & 3.1 & 2.9 & 2.5 \\
Peak overshoot (m) & 0.0050 & 0.0049 & 0.0047 & 0.0045 & 0.0041 & 0.0040 & 0.0036 \\
Peak undershoot (m) & 0.0042 & 0.0045 & 0.0046 & 0.0042 & 0.0042 & 0.0041 & 0.0040 \\
Noise (\%) & 10 & 10 & 5 & 5 & 5 & 4 & 1 \\
$e_{s s}(\%)$ & 0 & 0 & 0 & 0 & 0 & 0 & 0 \\
\hline
\end{tabular}




\section{Conclusion}

In this paper a novel control strategy BLQGC is proposed based on backstepping control approach. To justify its enhanced performance, it has been applied and tested to control the oscillation and the velocity of the suspension system in the vehicle. Initially, the VS system is modelled as a forth order state-space representation. Then the proposed control approach BLQGC is designed. In suggested BLQGC, state estimator based on the backstepping approach is utilized to enhance the control performance. The comparative results clearly reflect that the suggested BLQGC is arrive at better performance than the other control approaches such as PID, fuzzy, $\mathrm{NF}, \mathrm{LQR}, H_{\infty}$, and SM, with respect to stability, reliability, and robustness under various abnormal conditions and disturbances. The related better performance of the suggested approach (BLQGC) in terms of improved accuracy and stability, enhanced robustness, high noise elimination capability, and better capability to handle uncertainty justify its real time application.

The results imply that this type of robust control strategy can be implemented in real time using an integrated embedded system for control the oscillation and the velocity of the suspension system in the vehicle. The focus on the future studies will be on two major topics:

(1) Robust control methods

(2) Performance evaluation

However, during the pre-implementation stage the future direction in research will be for developing more accurate time dependent mathematical models to test and verify the practical scenario.

\section{Summary of this manuscript}

- Development of a SIMULINK model of a VS system.

- Design of a novel BLQGC to regulate the oscillation of the VS system within a stable range from -5 to $+5 \%$ of road disturbance and return to smooth ride within $5 \mathrm{~s}$.

- Evaluation of the control actions of the BLQGC under huge deviation of road impact.

- Comparative investigation to certify the better response of the BLQGC.

\section{Compliance with ethical standards}

Conflict of interest The author declares that he has no conflict of interest.

\section{References}

1. Wright PG (1984) The application of active suspension to high performance road vehicles. I Mech Eng Pap C 239:123

2. Fateh MM, Alavi SS (2009) Impedance control of an active suspension system. Mechatronics 19(1):134-140

3. Lin JS, Kanellakopoulos I (1997) Nonlinear design of active suspensions. IEEE Control Syst Mag 17(3):45-59

4. Alleyne A, Hedrick JK (1995) Nonlinear adaptive control of active suspensions. IEEE Trans Control Syst Technol 3(1):94-101

5. Esmailzadeh E, Taghirad HD (1998) Active vehicle suspensions with optimal state-feedback control. Int J Model Simul 18(3):228-238

6. Kumar MS (2008) Development of active suspension system for automobiles using PID controller. In: Proceedings of the world congress on engineering, WCE 2008, London, UK, pp 1472-1477

7. Talib MHA, Darns IZM (2013) Self-tuning PID controller for active suspension system with hydraulic actuator. In: 2013 IEEE symposium on computers and informatics (ISCI). IEEE, pp 86-91

8. Pekgökgöz RK, Gürel MA, Bilgehan M, Kısa M (2010) Active suspension of cars using fuzzy logic controller optimized by genetic algorithm. Int J Eng Appl Sci 2(4):27-37

9. Li H, Liu H, Gao H, Shi P (2012) Reliable fuzzy control for active suspension systems with actuator delay and fault. IEEE Trans Fuzzy Syst 20(2):342-357

10. Lin YJ, Lu YQ, Padovan J (1993) Fuzzy logic control of vehicle suspension systems. Int J Veh Des 14(5-6):457-470

11. Yoshimura T, Nakaminami K, Kurimoto M, Hino J (1999) Active suspension of passenger cars using linear and fuzzy-logic controls. Control Eng Pract 7(1):41-47

12. Yeh EC, Tsao YJ (1994) A fuzzy preview control scheme of active suspension for rough road. Int J Veh Des 15(1-2):166-180

13. Yue LJ, Tang CY, Li H (2008) Research on vehicle suspension systems based on fuzzy logic control. In: 2008 IEEE international conference on automation and logistics. IEEE, pp 1817-1821

14. Qiyao Y, Kongkang Z, Wenna Z, Xing X, Chunyuan Y (2008) FuzzyPID control on semi-active air suspension. Trans Chin Soc Agric Mach 39:24-29

15. Du H, Lam J, Sze KY (2003) Non-fragile output feedback $\mathrm{H} \infty$ vehicle suspension control using genetic algorithm. Eng Appl Artif Intell 16(7-8):667-680

16. Wang, Y. J. (2007). Analysis of vehicle suspension control using neural networks

17. Aldair AA, Wang WJ (2012) A neurofuzzy controller for full vehicle active suspension systems. J Vib Control 18(12):1837-1854

18. EIMadany MM, Abduljabbar ZS (1999) Linear quadratic Gaussian control of a quarter-car suspension. Veh Syst Dyn 32(6):479-497

19. Kaleemullah M, Faris WF, Hasbullah F (2011) Design of robust $\mathrm{H} \infty$, fuzzy and LQR controller for active suspension of a quarter car model. In: 2011 4th international conference on mechatronics (ICOM). IEEE, pp 1-6

20. Wang G, Zhou Z (2019) Design and implementation of $\mathrm{H}_{\infty}$ miscellaneous information feedback control for vehicle suspension system. Shock Vib. https://doi.org/10.1155/2019/3736402

21. Li H, Yu J, Hilton C, Liu H (2013) Adaptive sliding-mode control for nonlinear active suspension vehicle systems using T-S fuzzy approach. IEEE Trans Ind Electron 60(8):3328-3338 
22. Chen PC, Huang AC (2005) Adaptive sliding control of nonautonomous active suspension systems with time-varying loadings. J Sound Vib 282(3-5):1119-1135

23. Li W, Du H, Ning D, Li W (2019) Robust adaptive sliding mode PI control for active vehicle seat suspension systems. In: 2019 Chinese control and decision conference (CCDC). IEEE, pp 5403-5408

24. Khalil HK, Grizzle JW (2002) Nonlinear systems, vol 3. Prentice hall, Upper Saddle River

25. Patra AK, Rout PK (2018) Backstepping sliding mode Gaussian insulin injection control for blood glucose regulation in type I diabetes patient. J Dyn Syst Meas Control 140(9):091006
26. Patra AK, Mishra AK, Rout PK (2018) Backstepping model predictive controller for blood glucose regulation in type-I diabetes patient. IETE J Res 1(1):1-15

27. Patra AK, Biswal SS, Rout PK (2019) Backstepping linear quadratic Gaussian controller design for balancing an inverted pendulum. IETE J Res 1(1):1-15

Publisher's Note Springer Nature remains neutral with regard to jurisdictional claims in published maps and institutional affiliations. 